\title{
Características físico-hídricas de substratos formulados com lodo de esgoto na produção de mudas de acácia-negra
}

\author{
Physico-hydric characteristics in substrates formulated with sewage sludge in \\ the production of black wattle seedlings
}

\section{Alex Becker Monteiro', Adilson Luís Bamberg ${ }^{\mathrm{II}}$, Ivan dos Santos Pereira ${ }^{\mathrm{III}}$, Cristiane Mariliz Stöcker ${ }^{\mathrm{IV}}$, Luís Carlos Timm ${ }^{\mathrm{V}}$}

\begin{abstract}
Resumo
Avaliou-se o efeito da retenção de água de substratos para plantas formulados a partir de diferentes proporções de lodo de esgoto (LETE) solarizado, na produção de mudas de acácia-negra (Acacia mearnsii). O experimento foi conduzido em casa de vegetação e no Laboratório de Física do Solo da Embrapa Clima Temperado. O LETE utilizado foi coletado em leitos de secagem de uma Estação de Tratamento de Esgoto aeróbio de Rio Grande - RS. Os tratamentos consistiram de cinco doses de LETE solarizado, combinadas com cinza de casca de arroz e vermiculita em diferentes proporções (20:40:40, 30:35:35, 40:30:30, 50:25:25 e 60:20:20) e dois substratos comerciais (Beifort ${ }^{\circledR}$ S10 B e Mec Plant ${ }^{\circledR}$ ). O delineamento experimental foi de blocos casualizados. Foram avaliadas as características físico-hídricas: água facilmente disponível, água tamponante, água remanescente e capacidade de retenção de água. As variáveis de crescimento avaliadas foram: massa fresca da parte aérea e do sistema radicular. O substrato com $20 \%$ de LETE solarizado, $40 \%$ vermiculita e $40 \%$ cinza de casca de arroz apresentou a melhor condição físico-hídrica para o desenvolvimento de mudas de acácia-negra.
\end{abstract}

Palavras-chave: Acacia mearnsii; Biossólidos; Caracterização físico-hídrica de substratos

\begin{abstract}
This work evaluated the effect of water retention of plant substrates formulated with different proportions of solarized sewage sludge (SS) in the production of black wattle (Acacia mearnsii) seedlings. The study was conducted in a greenhouse and in the Laboratory of Soil Physics at the Lowland Experimental Station of Embrapa Clima Temperado. The SS was collected in drying beds at an aerobic sewage water treatment plant from Rio Grande, Rio Grande do Sul state. The treatments consisted of five doses of SS-solarized combined with rice husk ash and vermiculite in different proportions (20:40:40, 30:35:35, 40:30:30, 50:25:25 and 60:20:20) and two commercial substrates (controls) (Beifort ${ }^{\circledR}$ S10 B and Mec Plant ${ }^{\circledR}$ ). The experimental design was randomized blocks. The following physico-hydric atributes were determined easily available water, buffer water, remaining water and water retention capacity. The following variables were evaluated: dry mass of aerial parts and the dry mass of roots. The substrate with $20 \%$ of SS-solarized, $40 \%$ of vermiculite and $40 \%$ of rice husk ash presented the best physico-hydric condition for the development of black wattle seedlings.
\end{abstract}

Keywords: Acacia mearnsii; Biosolids; Physico-hydric characteristics of substrates

Tecnólogo em Irrigação e Drenagem, MSc, Doutorando no Programa de Pós-Graduação em Manejo e Conservação do Solo e da Água, Universidade Federal de Pelotas, Faculdade de Agronomia Eliseu Maciel, Departamento de Solos, Av. Eliseu Maciel, s/n, CEP 96050-500, Capão do Leão (RS), Brasil. alexbeckermonteiro@gmail.com (ORCID: 0000-0002- 1903-2454)

II Engenheiro Agrícola, Dr., Pesquisador A da Embrapa Clima Temperado, Rodovia BR 392, km 78, 9º Distrito - Monte Bonito, CEP 96010-971, Pelotas (RS), Brasil. adilson.bamberg@embrapa.br (ORCID: 0000-0002-3068-9836)

III Engenheiro Agrônomo, Dr., Pós-Doutorando no Programa de Pós-Graduação em Manejo e Conservação do Solo e da Água, Universidade Federal de Pelotas, Faculdade de Agronomia Eliseu Maciel, Departamento de Solos, Av. Eliseu Maciel, s/n, CEP 96050-500, Capão do Leão (RS), Brasil. ivanspereira@gmail.com (ORCID: 0000-0001-7431-780X)

Iv Engenheira Agrônoma, MSc., Doutoranda no Programa de Pós-Graduação em Sistemas de Produção Agrícola Familiar, Universidade Federal de Pelotas, Faculdade de Agronomia Eliseu Maciel, Departamento de Fitotecnia, Av. Eliseu Maciel, s/n, CEP 96050-500, Capão do Leão (RS), Brasil. crisstocker@yahoo.com.br (ORCID: 0000-0003-0961-5178)

Engenheiro Agrícola, Dr., Professor Associado IV da Universidade Federal de Pelotas, Faculdade de Agronomia Eliseu Maciel, Departamento de Engenharia Rural, Av. Eliseu Maciel, s/n, CEP 96050-500, Capão do Leão (RS), Brasil. lctimm@ufpel.edu.br (ORCID: 0000-0003-2916-8125) 


\section{Introdução}

Lodo de estação de tratamento de esgoto (LETE) é um resíduo semissólido gerado por plantas de tratamento de esgoto, originado após uma sequência de processos físicos, químicos e biológicos (LIU et al., 2018). A utilização ambientalmente correta do LETE tem sido preconizada, considerando diversas áreas, principalmente no que diz respeito ao seu uso agrícola (BONINI; ALVES; MONTANARI, 2015; KOŃCZAK; OLESZCZUK, 2018).

Neste contexto, a produção de mudas de espécies florestais pode ser uma das principais alternativas de uso do LETE. Segundo diversos autores, as características físicas e químicas apresentadas por substratos formulados a partir de LETE podem ser consideradas adequadas para o desenvolvimento de mudas de espécies florestais (CALDEIRA et al., 2012; FARIA et al., 2013; MONTEIRO et al., 2017).

A cultura da acácia-negra está entre as três espécies florestais de maior importância econômica cultivada no Rio Grande do Sul. A Acacia mearnsii é comumente cultivada no estado do Rio Grande do Sul, tendo grande importância socioeconômica, principalmente na região do Vale dos Sinos (SUYENAGA et al., 2015).

Contudo, embora os resultados de pesquisas venham demonstrando a eficiência agronômica do LETE na composição de substratos, sua utilização pode ser limitada pela presença de organismos patogênicos em níveis acima dos limites permitidos pela legislação (CONSELHO NACIONAL DO MEIO AMBIENTE, 2006). Desta forma, é importante a adoção de algum processo eficiente para a redução de agentes patogênicos. A solarização é uma alternativa eficiente para reduzir consideravelmente o seu teor de umidade, seu volume e proporcionar a desinfecção do material (MATHIOUDAKIS et al., 2013; SANTOS et al., 2015), adequando os LETEs aos limites estabelecidos pela resolução CONAMA 375/2006 (LIMA et al., 2009).

A constituição de um substrato formulado a partir de uma mistura de matérias-primas é muitas vezes um desafio, pois a qualidade final do produto está diretamente relacionada com a qualidade das matérias-primas que serão utilizadas para tal mistura, e da interação entre elas. De acordo com Fermino e Kämpf (2012), para que se obtenha sucesso na constituição de um substrato, o conhecimento prévio das propriedades físicas e químicas de cada mistura e, posteriormente, da interação destas com as sementes e mudas é crucial.

De acordo com a literatura, uma das principais características físicas a serem consideradas na caracterização dos substratos é a capacidade de retenção de água (DE BOODT; VERDONCK, 1972; FERMINO; KÄMPF, 2012; FERMINO, 2014; MONTEIRO et al., 2017). Dentro desse contexto, De Boodt e Verdonck (1972) definiram como pontos de referência para a construção da curva de retenção de água para substratos as tensões de $0,1,5$ e $10 \mathrm{kPa}$. A partir destas tensões é possível calcular a água facilmente disponível, água tamponante, água remanescente e também elaborar a curva de retenção de água dos substratos. De posse desses parâmetros físico-hídricos pode ser indicado o melhor manejo e a cultura que poderá melhor adaptar-se às características do substrato.

Neste contexto, o objetivo do trabalho foi avaliar o efeito da retenção de água de substratos para plantas formulados a partir de diferentes proporções de LETE solarizado sobre a produção de mudas de acácia-negra (Acacia mearnsii).

\section{Material e métodos}

O experimento foi conduzido durante os meses de agosto a outubro de 2016, em casa de vegetação, com temperatura controlada (15 a 30C), na Estação Experimental Terras Baixas da Embrapa Clima Temperado, município de Capão do Leão, Rio Grande do Sul (latitude 31 ${ }^{\circ} 49^{\prime} 13^{\prime \prime}$ $\mathrm{S}$, longitude $52^{\circ} 27^{\prime} 50^{\prime \prime} \mathrm{O}$ e altitude de $13 \mathrm{~m}$ ).

O lodo de estação de tratamento de esgoto (LETE) utilizado foi coletado em leito de secagem de uma Estação de Tratamento de Esgoto sanitário operada em processo aeróbio em Rio Grande, Rio Grande do Sul, pertencente à Companhia Riograndense de Saneamento - 
CORSAN. Após a coleta, o LETE foi solarizado, processo que consistiu da distribuição do mesmo em caixas de fibra de vidro, em camadas de $0,1 \mathrm{~m}$, acondicionadas no interior de uma estufa agrícola confeccionada em plástico transparente, com controle automatizado de aquecimento e aeração, permanecendo nesta condição por cerca de 60 dias, até que o LETE atingisse um teor de umidade inferior a 20\%. O LETE foi caracterizado quanto à presença e concentração de contaminantes, segundo a Resolução do CONAMA n 375/2006 (CONSELHO NACIONAL DO MEIO AMBIENTE, 2006), para verificar o pleno atendimento aos limites máximos admitidos.

Os substratos consistiram de cinco proporções de LETE solarizado, combinados com vermiculita e cinza de casca de arroz, além de dois substratos comerciais Beifort ${ }^{\circledR} \mathrm{S} 10 \mathrm{~B}$ e Mec Plant. Os substratos formulados foram: S1- 20\% LETE solarizado (LETE-sol) + 40\% vermiculita $(\mathrm{VER})+40 \%$ cinza de casca de arroz (CCA); S2- 30\% LETE-sol + 35\% VER + 35\% CCA; S3- 40\% LETE-sol + 30\% VER + 30\% CCA; S4- 50\% LETE-sol + 25\% VER + 25\% CCA; S5- 60\% LETE-sol + $20 \%$ VER + 20\% CCA; S6- substrato comercial 1 (Beifort ${ }^{\circledR}$ S10 B) e S7- substrato comercial 2 (Mec Plant ${ }^{\circledR}$.

Os substratos foram distribuídos em tubetes de polipropileno dispostos em suportes metálicos com capacidade de $55 \mathrm{~cm}^{3}$. As sementes de acácia-negra (Acacia mearnsii) foram doadas pela empresa TANAC, estas foram submetidas à quebra da dormência através da imersão das mesmas em água quente $\left(80^{\circ} \mathrm{C}\right)$ por 3 minutos (MARTINS-CORDER; BORGES; BORGES JUNIOR, 1999), obtendo-se $72 \%$ de germinação. A semeadura foi realizada no dia 25 de agosto de 2016, com duas sementes por tubete, sendo realizado posteriormente o desbaste deixandose uma planta por tubete. A irrigação ao longo do desenvolvimento das mudas foi realizada conforme a necessidade da cultura, através de aspersores.

Foram realizadas as seguintes avaliações das características físico-hídricas em amostras dos substratos: água facilmente disponível (AFD), água tamponante (AT), água remanescente (AR) e capacidade de retenção de água (CRA), de acordo com Fermino (2014). Para a análise de crescimento das plantas foram determinadas no dia 24 de novembro de 2016, 89 dias após a semeadura, a massa fresca da parte aérea (MFPA) (g) e do sistema radicular (MFSR) (g). Ambas foram determinadas em quatro plantas de cada parcela, sendo estas acondicionadas em sacos de papel e pesadas em balança analítica.

O delineamento experimental adotado foi de blocos casualizados, com três repetições de quatro plantas por parcela. Os resultados obtidos foram submetidos à análise de variância e para variáveis com efeito significativo (teste $\mathrm{F}, 5 \%$ de probabilidade), foi aplicado o teste de comparação de médias de Tukey a $5 \%$ de probabilidade.

\section{Resultados e discussão}

Os substratos testados influenciaram as características físico-hídricas avaliadas. Em relação à $\mathrm{AFD}$, houve decréscimo com o aumento da proporção de LETE-sol na formulação (Figura 1a). Nota-se que a AFD apresentou um modelo de resposta linear decrescente $\left(R^{2}=-0,68\right.$; $\mathrm{p}<0,01)$ em função do aumento das proporções de LETE-sol. Os valores de AFD observados no presente estudo ficaram entre $10,37 \%$ e $5,10 \%$, sendo inferiores à faixa indicada como ideal por De Boodt e Verdonck (1972), de 20 a 30\%. O substrato Comercial 1 apresentou AFD similar aos substratos com LETE-sol de pior desempenho, ou seja, baixo percentual de AFD (Figura 1a). Por outro lado, o substrato Comercial 2 teve resultado de AFD mais elevado, correspondente aos valores dos substratos com LETE-sol de melhor desempenho, que proporcionaram maior crescimento das mudas de acácia-negra (Figura 1a).

A AT também foi reduzida com o aumento das proporções de LETE-sol, ajustando-se a um modelo linear negativo $\left(\mathrm{R}^{2}=-0,86 ; \mathrm{p}<0,01\right)$ (Figura $1 \mathrm{~b}$ ). Os valores variaram de $5,78 \%$, no substrato com a menor proporção de LETE-sol (S1), à 0,95\% no substrato com a maior proporção (S5). Segundo De Boodt e Verdonck (1972), o valor ideal de AT fica em torno de 4 a 10\%, sendo que o valor obtido no substrato com $20 \%$ de LETE-sol está na faixa recomendada pelos autores. 
Para essa variável, o substrato Comercial 1, que proporcionou menor crescimento das mudas, apresentou nível de AT similar aos substratos formulados com LETE-sol de melhor desempenho, sendo o oposto verificado no Comercial 2.

Diferentemente das demais variáveis físico-hídricas (AFD e AT), a AR foi incrementada pelo aumento da proporção de LETE-sol, ajustando-se a um modelo linear positivo $\left(\mathrm{R}^{2}=0,88\right.$; $\mathrm{p}<0,01)$, ou seja, conforme aumentou-se a proporção de LETE-sol na formulação do substrato, maior foi a AR (Figura 1c). Os valores de AR aumentaram de 40,5\% no tratamento com a menor proporção de LETE-sol (S1) para 57,19\% naquele com a maior proporção (S5). Todas as formulações desenvolvidas com LETE-sol apresentaram valores de AR superiores aos recomendados por De Boodt e Verdonck (1972), que indicam como faixa adequada entre 20 e $30 \%$.

A CRA também foi incrementada pelo aumento da proporção de LETE-sol, ajustando-se um modelo linear positivo $\left(\mathrm{R}^{2}=0,82 ; \mathrm{p}<0,01\right)$, ou seja, conforme foi aumentada a proporção de LETE-sol nos substratos, maior foi a CRA (Figura 1d). Os substratos S1 e S2 (20 e 30\% de LETEsol) apresentaram valores de CRA dentro da faixa recomendada por De Boodt e Verdonck (1972) que indicam uma faixa entre 50 e 60\% (Figura 1d). Os demais substratos formulados com LETEsol (S3, S4 e S5) apresentaram CRA superior à faixa recomendada pelos autores. Já, os substratos comerciais apresentaram valores de CRA abaixo da faixa recomendada.

Em geral, o substrato com a menor proporção de LETE solarizado (S1) induziu o maior crescimento das mudas de acácia-negra. Esse resultado provavelmente ocorreu porque essa formulação apresentou os resultados de retenção de água mais próximos aos valores considerados ideais por De Boodt e Verdonck (1972), especialmente para AT e AR. Nota-se que conforme aumenta a proporção de LETE solarizado, os valores de AFD, AT, AR e CRA se distanciam dos valores ideais.

Apesar dos valores de AFD obtidos nesse estudo terem se situado abaixo da faixa ideal, aparentemente, isso não influenciou os resultados de fitomassa da parte aérea e de raízes das plantas de acácia obtidos neste estudo. Esse fato pode ser constatado pela ausência de correlação significativa da AFD com a MFPA e com a MFSR ( $r=0,51$ e $r=0,28$, respectivamente, não significativo a $p<0,05)$. Provavelmente, irrigações realizadas com frequência ao longo da condução do experimento tenham contornado os efeitos adversos dos baixos valores de AFD.

Em relação à AT, quantidade de água disponível entre as tensões de 5 e $10 \mathrm{kPa}$, pode-se dizer que os valores obtidos foram pouco expressivos, especialmente nas maiores doses de LETE solarizado $(\mathrm{AT}<2 \%)$, e por essa razão pouco devem ter contribuído para o maior ou menor crescimento das mudas.

Por outro lado, os valores da AR chegaram a $60 \%$ do total de água presente nos substratos. A AR está relacionada com a água retida a altas tensões, acima de $10 \mathrm{kPa}$, sendo considerada pouco acessível às plantas. Além disso, a AR representa a água presente nos microporos do substrato (SCIVITTARO et al., 2007), e por essa razão, altos valores de AR geralmente também significam baixos volumes de espaços de aeração. Por esse motivo, o montante de AR resultou em excesso de umidade limitando o desenvolvimento nos tratamentos com proporções elevadas de LETE solarizado. Isso poderia induzir a ideia de se reduzir a quantidade de LETE solarizado na formulação. Mas, reduzir demasiadamente a sua quantidade resultará em menor teor de nutrientes na formulação final. Alternativamente, podese recomendar a mistura de outros componentes com maior capacidade de aeração do substrato como, por exemplo, a casca de arroz carbonizada em substituição à cinza da casca de arroz.

Os substratos testados influenciaram significativamente o crescimento das mudas de acácianegra (Figura 2). O substrato com a menor proporção de LETE-sol (S1) proporcionou a maior massa fresca de parte aérea, enquanto os substratos S2, S3 e S5 tiveram as menores massas. Já os substratos S4 e S7 tiveram resultados intermediários e não diferiram dos demais. Em relação à massa fresca de sistema radicular, o substrato com a menor proporção de LETE-sol (S1) e o substrato comercial Mec Plant ${ }^{\circledR}$ (S7) apresentaram os maiores valores. Por outro lado, os substratos S2, S3 e S5 tiveram as menores massas de sistema radicular, enquanto que os substratos S4 e S6 tiveram resultados intermediários e que não diferiram dos demais substratos avaliados (Figura 2). 
Figura 1 - Água facilmente disponível (AFD), água tamponante (AT), água remanescente (AR) e capacidade de retenção de água (CRA) em substratos com LETE solarizado (a, b, c e d, respectivamente). S1- 20\% LETE solarizado (LETE sol) + $40 \%$ vermiculita (VER) + $40 \%$ cinza de casca de arroz (CCA); S2$30 \%$ LETE sol + 35\% VER + 35\% CCA; S3- 40\% LETE sol + 30\% VER + 30\% CCA; S4- 50\% LETE sol + 25\% VER + 25\% CCA; S5- 60\% LETE sol + 20\% VER + 20\% CCA; S6- substrato comercial 1 (Beifort ${ }^{\circledR}$ S10 B); S7- substrato comercial 2 (Mec Plant $\left.{ }^{\circ}\right) .{ }^{* *}$ significativo a 1\%; Referência - De Boodt e Verdonck (1972).

Figure 1 - Water easily available (WEA), water buffer (WB), remaining water (WR) and water retention capacity (WRC) in substrates with SS solarized (a, b, c and d, respectively). S1- 20\% SS solarized (SS sol) + $40 \%$ vermiculite (VER) + 40\% rice husk ash (RHA); S2- 30\% SS sol + 35\% VER + 35\% RHA; S3- $40 \%$ SS sol + 30\% VER + 30\% RHA; S4- 50\% SS sol + 25\% VER + 25\% RHA; S5- 60\% SS sol + 20\% VER + 20\% RHA; S6- commercial substrate 1 Beifort ${ }^{\circledR}$ S10 B; S7- commercial substrate 2 Mac Plant ${ }^{\circledR}{ }^{~}{ }^{* *}$ significance of $1 \%$; Reference - De Boodt e Verdonck (1972).

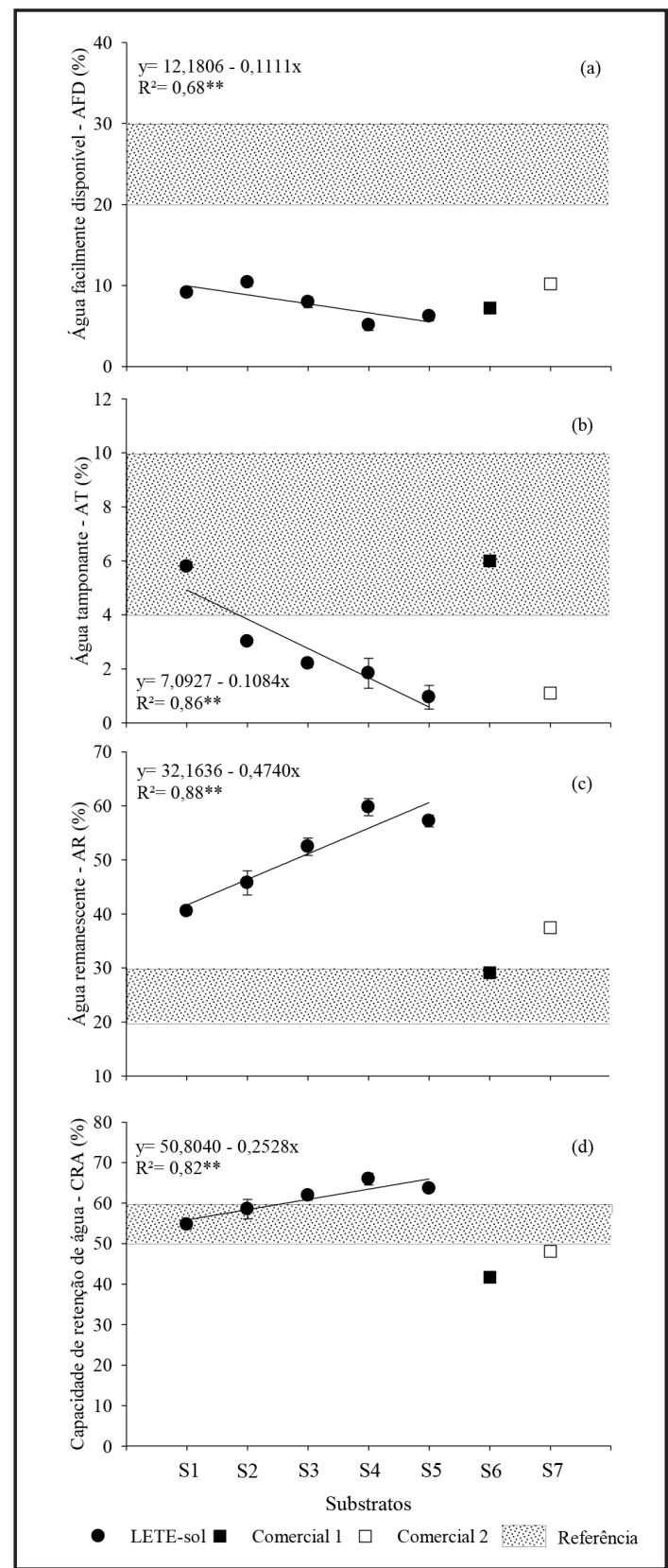

Fonte: Autores (2019)

Source: Authors (2019) 
Figura 2 - Massa fresca de parte aérea (MFPA) e massa fresca do sistema radicular (MFSR) de mudas de acácia-negra cultivadas com diferentes substratos formulados à base de lodo de esgoto sanitário. * Médias seguidas da mesma letra, não diferem pelo teste de Tukey a $5 \%$ de probabilidade. S1- 20\% LETE solarizado (LETE sol) + 40\% vermiculita (VER) + 40\% cinza de casca de arroz (CCA); S2- 30\% LETE sol + 35\% VER + 35\% CCA; S3- 40\% LETE sol + 30\% VER + 30\% CCA; S4- 50\% LETE sol + 25\% VER + 25\% CCA; S5- 60\% LETE sol + 20\% VER + 20\% CCA; S6- substrato comercial 1 (Beifort ${ }^{\circledR}$ S10 B) e S7- substrato comercial 2 (Mec Plant ${ }^{\circledR}$ ).

Figure 2 - Shoot fresh mass and root fresh mass of black wattle seedlings grown on different substrates with sewage sludge. ${ }^{*}$ Means followed by the same lowercase letter do not differ by the Tukey test at $5 \%$ probability. S1- $20 \%$ SS solarized (SS sol) $+40 \%$ vermiculite (VER) $+40 \%$ rice husk ash (RHA); S2- 30\% SS sol + 35\% VER + 35\% RHA; S3- 40\% SS sol + 30\% VER + 30\% RHA; S4- 50\% SS sol + 25\% VER + 25\% RHA; S5- 60\% SS sol + 20\% VER + 20\% RHA; S6- commercial substrate 1

Beifort ${ }^{\circledR}$ S10 B; S7- commercial substrate 2 Mac Plant ${ }^{\circledR}$.

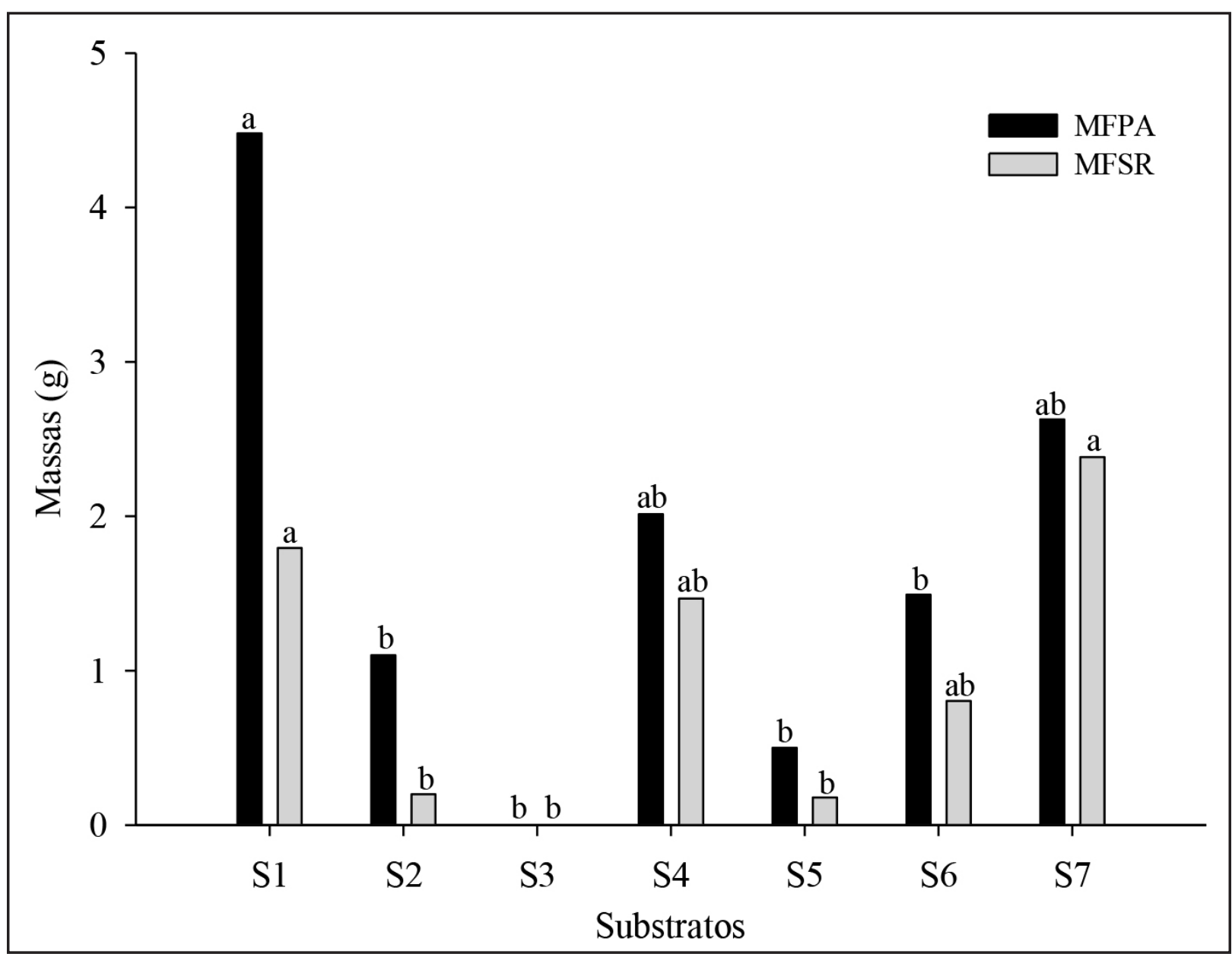

Fonte: Autores (2019)

Source: Authors (2019)

Caldeira et al. (2014) avaliando diferentes composições de substratos contendo lodo de esgoto associado à casca de arroz in natura, vermiculita e ao composto orgânico (CO) na produção de mudas de Acacia mangium, observaram que os melhores resultados foram obtidos com uma proporção de $40 \%$ de lodo de esgoto $+60 \%$ de composto orgânico. Pois, de acordo com os autores foi a mistura que proporcionou as melhores características avaliadas e, portanto, a mais indicada para a produção de mudas de Acacia mangium. 
Também trabalhando com uma espécie florestal, Scheer, Carneiro e Santos (2010) avaliaram a eficiência de um substrato à base de resíduos de poda de árvores trituradas (70\%) e compostadas com LETE (30\%), e os resultados foram semelhantes aos obtidos com o uso do substrato comercial, indicando que o substrato à base de LETE é uma boa alternativa para a produção de mudas de Parapiptadenia rigida. Caldeira et al. (2012), estudando o LETE combinado a solo como substrato para produção de mudas de Toona ciliata var. australis, verificaram que os melhores resultados foram obtidos com a utilização de 70 a 100\% de LETE, sendo o LETE considerado adequado para o crescimento de mudas desta espécie e uma alternativa viável de disposição final desse resíduo.

Através dos resultados obtidos no presente estudo e da literatura, pode-se observar que o LETE é uma matéria-prima interessante para compor substratos para a produção de mudas de espécies florestais. E que a sua proporção dentro do substrato irá variar, principalmente, de acordo com as outras matérias-primas que serão utilizadas.

\section{Conclusão}

A inclusão do LETE solarizado proporcionou substratos que resultaram em eficiência agronômica similar ou superior aos substratos comerciais avaliados para a cultura da acácianegra, principalmente nas formulações com menores proporções de LETE solarizado.

O substrato com $20 \%$ de LETE solarizado, $40 \%$ vermiculita e $40 \%$ cinza de casca de arroz apresentou a melhor condição físico-hídrica para o desenvolvimento de mudas de acácia-negra.

\section{Referencias}

BONINI, C. S. B.; ALVES, M. C.; MONTANARI, R. Lodo de esgoto e adubação mineral na recuperação de atributos químicos de solo degradado. Revista Brasileira de Engenharia Agrícola e Ambiental, Campina Grande, v. 19, n. 4, p. 388-393, 2015.

CALDEIRA, M. V. W. et al. Biossólido como substrato para a produção de mudas de Toona ciliata var. australis. Revista Árvore, Viçosa, MG, v. 36, n. 6, p. 1009-1017, 2012.

CALDEIRA, M. V. W. et al. Lodo de esgoto como componente de substrato para produção de mudas de Acacia mangium Wild. Comunicata Scientiae, Piauí, v. 5, n. 1, p. 34-43, 2014.

CONSELHO NACIONAL DO MEIO AMBIENTE (Brasil). Resolução Conama n. 375, de 29 de agosto de 2006. Diário Oficial da República Federativa do Brasil, Brasília, DF, 2006.

DE BOODT, M.; VERDONCK, O. The physical properties of the substrates in: Floriculture. Acta Horticulturae, Leuven, v. 26, n. 1, p. 37-44, 1972.

FARIA, J. C. T. et al. Substratos à base de lodo de esgoto na produção de mudas de Senna alata. Comunicata Scientiae, Piauí, v. 4, n. 4, p. 342-351, 2013.

FERMINO, M. H.; KÄMPF, A. N. Densidade de substratos dependendo dos métodos de análise e níveis de umidade. Horticultura Brasileira, Brasília, DF, v. 30, n. 1, p. 75-79, 2012.

FERMINO, M. H. Substratos: composição, caracterização e métodos de análise. Guaíba: Agrolivros, 2014.

KOŃCZAK, M.; OLESZCZUK, P. Application of biochar to sewage sludge reduces toxicity and improve organisms growth in sewage sludge-amended soil in long term field experimente. Science of the Total Environment, Amsterdam, v. 625, p. 8-15, 2018.

LIMA, M. R. P. et al. Avaliação da qualidade do lodo de esgoto quando submetido à secagem/ 
higienização em estufa agrícola. Revista AIDIS de Ingeniería en Ciencias Ambientales: investigación, desarrollo y prácticas, México, v. 2, n. 1, p. 41-48, 2009.

LIU, X. et al. Pyrolysis and subsequent direct combustion of pyrolytic gases for sewage sludge treatment in China. Applied Thermal Engineering, Amsterdam, v. 128, p. 464-470, 2018.

MARTINS-CORDER, M. P.; BORGES, R. Z.; BORGES JUNIOR, N. Fotoperiodismo e quebra de dormência em sementes de Acácia-Negra (Accacia mearnsii De Wild.). Ciência Florestal, Santa Maria, v. 9, n. 1, p. 71-77, 1999.

MATHIOUDAKIS, V. L. et al. Sewage sludge solar drying: experiences from the first pilot-scale application in Greece. Drying Technology: An International Journal, New York, v. 31, n. 5, p. 519-526, 2013.

MONTEIRO, A. B. et al. Substratos a base de lodo de esgoto solarizado e pirolizado para produção de mudas de alface. Revista Congrega Urcamp, Bagé, p. 13, 2017.

SANTOS, A. G. V. A. et al. Secagem do biossólido em uma estufa agrícola. Revista de Iniciação Científica, Tecnológica e Artística, São Paulo, p. 13, 2015.

SCHEER, M. B.; CARNEIRO, C.; SANTOS, K. G. dos. Substratos à base de esgoto compostado na produção de mudas de Parapiptadenia rigida (Benth.) Brenan. Scientia Forestalis, Piracicaba, v. 38 , n. 88 , p. 637-644, 2010.

SCIVITTARO, W. B. et al. Caracterização física de substratos elaborados a partir de resíduos agroindustriais. Pelotas: EMBRAPA Clima Temperado, 2007. (Boletim de Pesquisa e Desenvolvimento).

SUYENAGA, E. S. et al. Acácia-negra: potenciais usos de reaproveitamento. Revista Conhecimento Online, [s. l.], v. 1, p. 2-7, 2015. 